\title{
DESAFIOS PARA A MENSURAÇÃO DE ECOSSISTEMAS DE INOVAÇÃO E DE EMPREENDEDORISMO NO BRASIL
}

Renata Lèbre La Rovere ${ }^{1}$

Guilherme Santos ${ }^{1}$

Bianca Louzada Xavier Vasconcellos ${ }^{1}$

${ }^{1}$ UFRJ/Instituto de Economia/Programa de Pós-Graduação em Políticas Públicas, Estratégias e Desenvolvimento (PPED) 


\section{DESAFIOS PARA A MENSURAÇÃO DE ECOSSISTEMAS DE INOVAÇÃO E DE EMPREENDEDORISMO NO BRASIL}

Resumo: A literatura sobre o papel dos empreendedores no desenvolvimento econômico tem sugerido que as interações provocadas pela atividade empreendedora podem ser analisadas através do conceito de ecossistemas de empreendedorismo. Ao mesmo tempo, a literatura sobre ecossistemas de inovação tem mostrado as diferentes dimensões deste tipo de ecossistema, que requerem diferentes métricas. Neste sentido, a proposição de métricas de ecossistemas de inovação e de empreendedorismo é fundamental para o desenho de políticas públicas de apoio à inovação, sobretudo aquelas políticas dirigidas a empreendedores e a pequenas empresas. $\mathrm{O}$ objetivo deste artigo, portanto, é elencar algumas destas métricas no caso brasileiro e discutir suas limitações. Para tanto, faremos uma breve discussão a respeito dos conceitos de ecossistema de inovação e ecossistema de empreendedorismo, apontando suas diferenças e complementaridades. Em seguida, apresentaremos possíveis métricas de ecossistemas de inovação e de ecossistemas de empreendedorismo no Brasil. A conclusão trará uma síntese dos resultados encontrados.

Palavras-chave: Ecossistemas de Inovação. Ecossistemas de Empreendedorismo. Métricas.

\section{$1 \quad$ Introdução}

A literatura sobre o papel dos empreendedores no desenvolvimento econômico tem sugerido que as interações provocadas pela atividade empreendedora podem ser analisadas através do conceito de ecossistemas de empreendedorismo (Spigel e Harrison, 2017; Nicotra et al., 2017; Neumeyer e Santos, 2018; O’Connor et al., 2018; Liguori et al., 2018; Stam, 2018). Ao mesmo tempo, a literatura sobre ecossistemas de inovação (Jackson, 2011; Gomes et al., 2016; Oh et al. 2016) tem mostrado as diferentes dimensões deste tipo de ecossistema, que requerem diferentes métricas (Carayannis et al., 2018).

Existem diferenças entre as duas abordagens. Enquanto o conceito de ecossistemas de inovação foca nas empresas como enquanto atores da inovação, o conceito de ecossistema de empreendedorismo foca nas habilidades individuais para inovar que caracterizam a atividade empreendedora (Xu e Maas, 2019).

Entretanto, os conceitos de ecossistemas de inovação e de ecossistemas de empreendedorismo não devem ser vistos como concorrentes e sim como complementares. Xu e Maas (2019) elencam uma lista de princípios comuns das duas abordagens que deveriam informar as políticas públicas de apoio ao empreendedorismo e à inovação. Esses princípios são: escutar as necessidades locais, pois cada contexto é único; ter visão de longo prazo, uma vez que os ecossistemas levam tempo para se desenvolver; trabalhar coletivamente, envolvendo o setor público e o privado; agir de forma responsiva, ou seja, ter em conta o fato de que os ecossistemas evoluem ao longo do tempo; e compartilhar histórias motivacionais para dar confiança às partes interessadas. Portanto, na visão destes autores, o Estado pode atuar tanto como alimentador de um ecossistema como stakeholder deste ecossistema. 
Partindo-se do pressuposto que cada contexto é único e que ecossistemas levam tempo para se desenvolver, a proposição de métricas de ecossistemas de inovação e de empreendedorismo é fundamental para o desenho de políticas públicas de apoio à inovação, sobretudo aquelas políticas dirigidas a empreendedores e a pequenas empresas. O objetivo deste artigo é elencar algumas destas métricas no caso brasileiro e discutir suas limitações. Para atingir este objetivo, a seção 2 fará uma breve discussão a respeito dos conceitos de ecossistema de inovação e ecossistema de empreendedorismo, apontando suas diferenças e complementaridades. A seção 3 apresentará possíveis métricas de ecossistemas de inovação e de ecossistemas de empreendedorismo no Brasil. A seção final trará as conclusões e recomendações para pesquisas futuras.

\section{Ecossistemas de Inovação e Ecossistemas de Empreendedorismo}

\subsection{Ecossistemas de Inovação}

O conceito de ecossistema de inovação, segundo Xu e Mass (2019) está relacionado ao conceito de sistemas de inovação, proposto por Lundvall (1992) e ao conceito de sistemas nacionais de inovação, proposto por Freeman (1987). A partir dos trabalhos seminais destes dois autores, vários autores da corrente econômica evolucionária passaram a analisar sistemas de inovação, em escala local, regional e nacional. Os evolucionários, como observado por Nelson (2018), se distinguem das demais correntes do pensamento econômico por colocar a mudança contínua, guiada na maior parte do tempo pela inovação, como centro da teoria econômica. Sua fonte reside nos trabalhos de Schumpeter, para quem o capitalismo é caracterizado não por ciclos de crescimento que tendem ao equilíbrio, e sim por ciclos de mudança intercalados por períodos de relativa estabilidade.

Ao analisar os processos de mudança, os autores evolucionários costumam utilizar analogias entre o sistema econômico e o sistema biológico. Para eles, as firmas detêm recursos que se mostram mais ou menos adequados ao processo competitivo resultante da interação entre elas e o mercado. As firmas mais bem-sucedidas tendem a sobreviver por mais tempo e replicar suas práticas através de rotinas, do mesmo modo que organismos mais aptos a sobreviver em determinados ambientes tendem a sobreviver por mais tempo e replicar suas características através de mecanismos de transmissão genética. A analogia, porém, deve ser utilizada com cuidado: enquanto a evolução biológica é um processo "cego", determinado pelas próprias interações entre organismos (Dawkins, 2007), a evolução econômica se caracteriza por intencionalidade. Como observa Nelson (2018, p.7):

\footnotetext{
A principal difference between economic evolution and biological evolution is that economic actors generally are able to choose what they are doing and how they are doing it, and have the capability to learn not only from their own experience but from available information about alternatives.
}

Ao propor o conceito de sistemas de inovação, os autores evolucionários chamam atenção para a importância do contexto onde a firma está atuando, e enfatizam a importância de se entender os processos de aprendizado que irão fomentar a atividade inovadora.

O conceito de ecossistema de inovação reforça o aspecto sistêmico da atividade inovadora das empresas, enfatizando o aspecto da coevolução entre os agentes que caracteriza qualquer processo sistêmico (Xu e Maas, 2019). Ele é uma adaptação do conceito proposto por Moore (1993) de ecossistema de negócios. Um ecossistema de inovação modela a dinâmica econômica 
das interações entre os agentes econômicos que desenvolvem tecnologia e inovações. Por atores entende-se não apenas o capital físico e humano engajado em inovação como também as instituições que participam do ecossistema (Jackson, 2011).

Entretanto, o conceito é considerado por diversos autores (Oh et al., 2016; Gomes et al., 2016), como um conceito com pouca fundamentação: de um lado, a analogia entre o mundo das firmas e o mundo dos organismos deve ser feita com ressalvas, como já visto. Por outro lado, os textos sobre ecossistemas de inovação não estabelecem uma distinção clara entre ecossistema e sistema de inovação. Apesar disso, o conceito vem atraindo interesse crescente tanto de acadêmicos quanto de gestores públicos envolvidos com a atividade inovadora. Não por acaso, o artigo de Jackson (2011), que trabalhava na National Science Foundation ao escrevê-lo, é lembrado quando se recupera o conceito. A difusão do conceito entre formuladores de política levou alguns autores a se perguntar como desenhar, construir e operar um ecossistema de inovação favorável. As respostas a estas perguntas, porém, frequentemente se desenvolvem recuperando uma visão linear da atividade inovadora que conflita com o próprio conceito (Wallner e Menrad, 2011). Oh et al. (2016) observam que, ao considerar ser possível desenhar um ecossistema, alguns autores realizam análises teleológicas buscando identificar o propósito do ecossistema, o que contradiz a ideia de que o ecossistema se molda a partir de interações. $\mathrm{O}$ desafio maior, na visão dos autores, reside justamente na dificuldade de estabelecer métricas para analisar um ecossistema de inovação.

Para escapar das armadilhas relacionadas ao conceito, que ainda está em construção, cabe entender melhor o que a sua análise envolve. Para Oh et al. (2016), os traços distintivos de publicações recentes que trabalham com o conceito de ecossistema de inovação são: explicitação do caráter sistêmico da atividade inovadora; reconhecimento da importância das tecnologias de informação e de comunicação no estabelecimento de redes entre os atores do sistema; inovação aberta; capacidade de imitação dos atores do sistema; ênfase nos papéis diferenciados que organizações e firmas assumem no sistema; importância das forças de mercado.

Jackson (2011), por sua vez, ressalta que num mesmo ecossistema convivem duas "economias" (ou subsistemas econômicos), que se relacionam fracamente: a economia da pesquisa (acadêmica) e a economia comercial (de mercado). A estes aspectos, Wallner e Menrad (2011) acrescentam o aspecto da cultura estabelecendo uma distinção entre inovação e capacidade inovadora: a inovação é a manifestação da capacidade inovadora, que por sua vez é fortemente influenciada por traços culturais. Como exemplos de traços culturais que fazem a diferença na capacidade inovadora, os autores citam a aversão ao risco, a percepção de fracasso como aprendizado (e não como derrota), a disposição em compartilhar informações e conhecimento e a tolerância à diversidade de ideias e pessoas.

\subsection{Ecossistemas de Empreendedorismo}

A Abordagem de Ecossistemas de Empreendedorismo (AEE), fruto da conjugação de pesquisas recentes nas áreas de estudos sobre empreendedorismo, geografia econômica, economia urbana, e economia do empreendedorismo, busca compreender de que forma os contextos urbanos e regionais afetam o empreendedorismo ambicioso $^{1}$ (Stam e Spigel, 2016).

${ }^{1}$ Ambitious Entrepreneurship, no original em inglês. Para mais detalhes ver Baumol (1990). 
Os trabalhos seminais de Pennings (1982), Dubini (1989), Van de Ven (1993) e Bahrami e Evans (1995) desenvolveram o conceito de "ambiente (ou ecossistema) empreendedor" com intuito de explicar a influência dos fatores sociais e econômicos das regiões sobre o processo empreendedor. Em síntese, o contexto importa. Não obstante, na AEE o empreendedor é o ponto focal da análise, em detrimento da empresa. Neste prisma, o foco da pesquisa sobre Ecossistemas de Empreendedorismo é baseada fortemente no empreendedor e nas startups, em detrimento de empresas maiores e mais bem estabelecidas, ou de pequenas e médias empresas de baixo crescimento.

Além dos conhecimentos relacionados ao know-how técnico necessário para desenvolver novos produtos e tecnologias, e ao funcionamento do mercado, a Abordagem de Ecossistemas de Empreendedorismo também ressalta o conhecimento sobre o processo empreendedor em si. Esta modalidade inclui o conhecimento sobre: os desafios que os empreendedores enfrentam enquanto desenvolvem seus negócios; como desenhar planos de negócios e pitches de ideias para investidores anjos e capitalistas de risco; e como superar a questão da falta de experiência quando estiverem trabalhando com potenciais clientes e fornecedores. Assim, a mentoria e o networking entre empreendedores são elementos críticos para o compartilhamento de conhecimento empreendedor dentro de um ecossistema (Lafuente et al. 2007).

Cabe ressaltar que, diferente de outros conceitos - p. ex. distritos industriais, clusters, learning regions e Sistemas Regionais de Inovação -, a Abordagem de Ecossistemas de Empreendedorismo não vê o empreendedorismo somente como resultado do sistema, mas também considera a importância dos empreendedores enquanto players centrais (líderes) na criação do sistema e na manutenção de sua vitalidade (Feldman, 2014). Esta concepção tem como consequência a diminuição do papel do governo, que é visto mais como um "alimentador" ("feeder") do ecossistema do que como um "líder" (“leader") (Feld, 2012).

Com base nas diversas contribuições encontradas na literatura, Mason e Brown (2014) sintetizam a definição de um Ecossistema Empreendedor da seguinte forma:

\begin{abstract}
Um conjunto interconectado de atores empreendedores (tanto potenciais quanto existentes), organizações empreendedoras (e.g. firmas, capitalistas de risco, investidores anjo, bancos), instituições (universidades, agências do setor público, instituições financeiras), e processos empreendedores (e.g. taxa de nascimento de negócios, número de firmas de alto crescimento, níveis de empreendedorismo de alto impacto, número de empreendedores seriais, níveis de ambição empreendedora), que de maneira formal e informal criam uma amálgama capaz de conectar, mediar, e governar a performance dentro de um ambiente empreendedor local. (Mason e Brown, 2014, p.5).
\end{abstract}

Neste prisma, Isenberg (2010) aponta que os gestores públicos que buscam fomentar um Ecossistema Empreendedor em sua região devem enfatizar o papel das condições locais e de processos bottom-up; enfatizar o empreendedorismo ambicioso, ou seja, favorecer os empreendedores de alto impacto; e focar nas instituições, sobretudo no estímulo ao desenvolvimento de uma cultura empreendedora e no estabelecimento de um framework legal, burocrático e regulatório propício ao desenvolvimento do empreendedorismo. Baseado nisso, o autor identificou seis domínios distintos dentro de um Ecossistema de Empreendedorismo: uma cultura propícia, políticas públicas de apoio, disponibilidade de financiamento apropriada, qualidade do capital humano, mercados abertos, e um conjunto de instituições de suporte.

Stam e Spigel (2016), por seu turno, distinguem os elementos de um Ecossistema de Empreendedorismo em duas categorias: condições de framework e condições sistêmicas. As 
condições de framework incluem as condições sociais (instituições formais e informais) e as condições físicas que estimulam e restringem a interação humana; ao passo que as condições sistêmicas estão no coração do ecossistema: redes de empreendedores, liderança, financiamento, talentos, conhecimento e serviços de apoio. A presença destes elementos e a interação entre eles são cruciais para o sucesso do ecossistema. O Quadro 1 destaca as principais semelhanças e diferenças entre ambas as abordagens.

\section{Quadro 1: Principais semelhanças e diferenças entre as Abordagens de Ecossistemas de} Inovação e Ecossistemas de Empreendedorismo

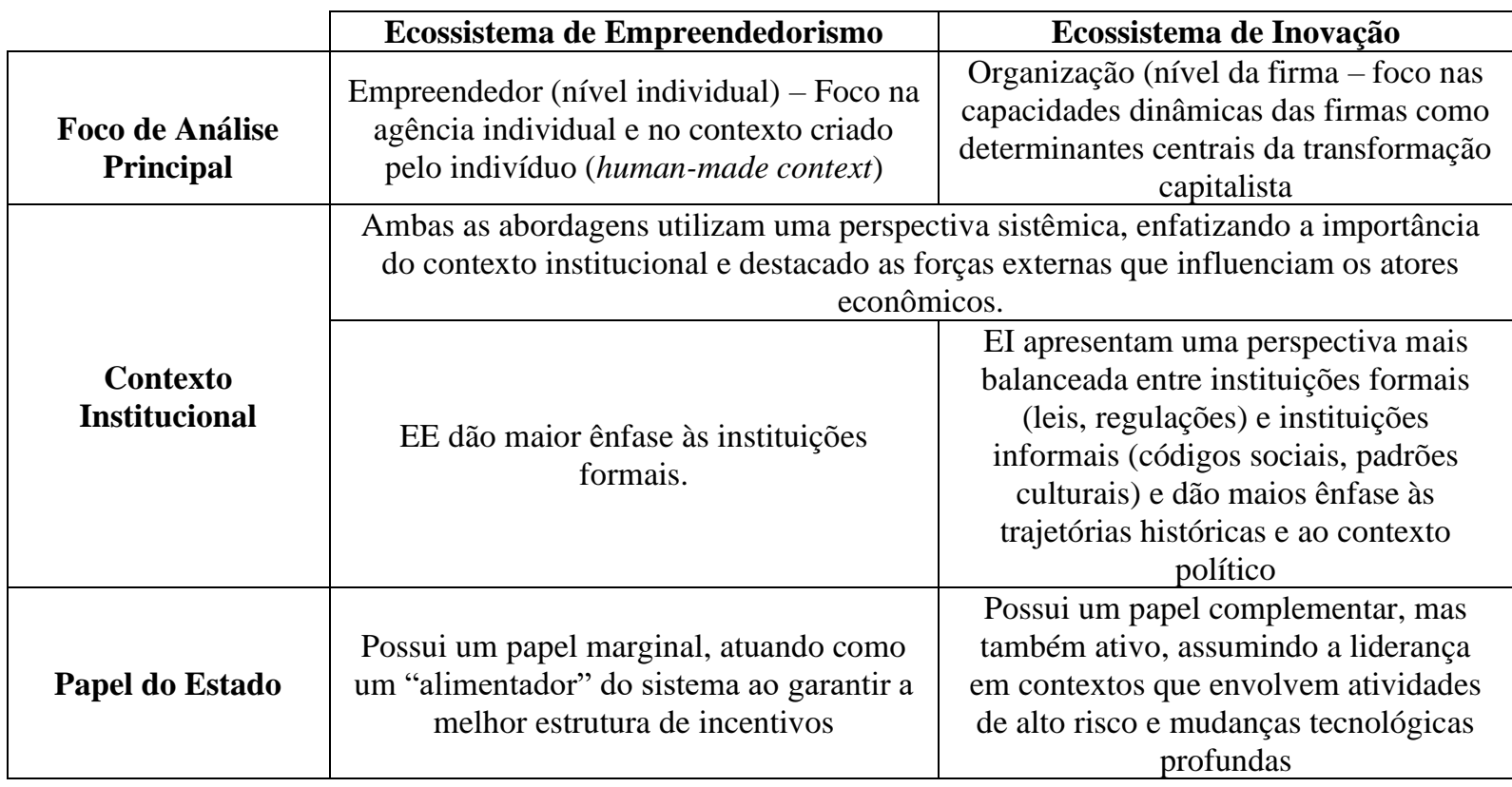

Fonte: Elaboração Própria (2019)

\section{Métricas para caracterizar Ecossistemas de Inovação e Ecossistemas de Empreendedorismo}

Como apontado acima, existem semelhanças e diferenças entre as abordagens de ecossistemas de inovação e de ecossistemas de empreendedorismo. Há autores que propõem métricas para ecossistemas de inovação que também podem ser utilizadas para mensurar ecossistemas de empreendedorismo. Ao mesmo tempo, existem métricas específicas que podem ser aplicadas para ecossistemas de inovação e para ecossistemas de empreendedorismo. Iremos detalhar estas métricas a seguir.

\subsection{Ecossistemas de inovação}

No que se refere aos ecossistemas de inovação, há métricas em nível nacional e métricas em nível regional. Em nível nacional, há vários indicadores quantitativos consolidados na literatura, a saber: remuneração da mão de obra com educação superior, número de mestres e doutores por cem mil habitantes, gastos em pesquisa e desenvolvimento (P\&D) como porcentagem do $\mathrm{PIB}$, número de licenciamentos, número de patentes, número de empresas de alto crescimento, parcela de venture capital no financiamento a empresas de base tecnológica. Além disso, há indicadores oficiais que constam de pesquisas por amostra que identificam: a 
capacidade inovadora de diversos setores industriais de um determinado país, os investimentos realizados em inovação, o percentual de empresas que estabelecem redes de cooperação e várias outras informações relevantes.

A geração de novo conhecimento que flui nos ecossistemas de inovação pode ter como proxy tanto os investimentos públicos quanto privados em Ciência e Tecnologia (Stam, 2018). O mapeamento das competências científicas locais pode ser feito a partir da identificação das Instituições Científico-Tecnológicas (ICTs) presentes no território, bem como dos grupos de pesquisa e de suas respectivas áreas (Nicotra et al., 2017). A identificação de redes entre grupos de pesquisa e empresas é também uma métrica possível da capacidade inovadora de regiões ou países (Urti, 2017).

Outra medida possível da capacidade de inovação das empresas é medir a complexidade do produto, definida como o montante de conhecimento produtivo, seu uso e seu contínuo aprimoramento numa determinada economia, ou seja, a capacidade da empresa de elaboração de produtos intensivos em conhecimento e, consequentemente, de formar mercados para os produtos e redes para a difusão deste conhecimento. O conceito parte do pressuposto de que o conhecimento coletivo não depende exclusivamente do conhecimento individual, mas sim da combinação de uma diversidade de conhecimentos de uma sociedade, que é capaz de criar novos produtos, através de complexas redes de interação. De acordo com Hausmann et al. (2013) os produtos são veículos para o conhecimento. O conceito de complexidade proposto por Haussmann et al. (2013), concebido originalmente para mensurações em nível nacional, também pode ser aplicado a cidades, regiões e estados de um mesmo país, sendo assim uma métrica possível para ecossistemas de inovação, uma vez que permite medir as redes de interação de conhecimento que circulam neste ecossistema. O indicador de complexidade é obtido a partir da metodologia do "Product Space" (ou espaço de produtos) desenvolvida por estes autores, na qual os produtos exportados pelos lugares são agrupados em redes, diferenciados por setores e exibidos num gráfico cujas cores demostram o quanto cada produto é mais ou menos complexo.

O 'Product Space' (...) permite entender, a partir da pauta de exportação dos países, quais os conhecimentos produtivos que cada nação possui. Essas capacidades ou habilidades são compostas por diversos fatores, tais como: capital, trabalho, tecnologia, instituições, infraestrutura, existência de relações sociais, dentre outros. Quanto mais conhecimento produtivo uma determinada localidade possui, maior o número de produtos que ela pode produzir e exportar, e mais complexos são esses produtos. (Plataforma Data Viva, 2019)

Uma métrica complementar à anterior é o indicador de entropia, que indica a diversidade ou variedade setorial. O conceito de entropia surgiu primeiro no campo da física para estudos dedicados à termodinâmica, depois se tornou uma métrica de sistemas complexos na economia (Furtado et al., 2015). De acordo com Vieira (2013) a entropia é uma medida de heterogeneidade, quanto maior a heterogeneidade mais intensa é a transformação de um sistema, portanto quanto maior a entropia, maior a complexidade de um fenômeno.

Na geografia econômica este indicador também vem sendo utilizado para mensurar a variedade setorial e seus efeitos sobre as regiões e a inovação, mas especificamente no entendimento do conceito de variedade relacionada (Frenken et al., 2007; Boschma e Iammarino, 2009; Castaldi et al., 2015). A variedade relacionada pode ser definida como o conjunto de setores que compartilham proximidades em termos de competências (Frenken, 2006). 
Segundo Frenken et al. (2007), o conceito de variedade relacionada expressa a ideia de que alguns setores são mais relacionados que outros, e por isso geram relativamente mais externalidades de Jacobs ${ }^{2}$. O grau de entropia é utilizado para examinar empiricamente os efeitos de variedade relacionada ou não-relacionada em uma região e seus setores industriais.

A principal vantagem da utilização do indicador de entropia, e a razão para ser usado, é o contexto de diversificação, pois esse indicador pode ser decomposto em cada nível setorial. Isso ajuda na compreensão da variedade relacionada (em nível de cinco dígitos da classificação de atividades econômicas) e variedade não-relacionada (em nível de dois dígitos da classificação de atividades econômicas) (Frenken et al., 2007). Já a principal vantagem do indicador de complexidade econômica é a utilização de uma metodologia mais sofisticada, no campo dos estudos de redes. Os indicadores podem assim se complementar para gerar um "retrato" da estrutura produtiva dos lugares.

Há também indicadores de Bases de Conhecimento. Asheim et al. (2011) propõem que o conhecimento pode ser dividido em três bases: analítica, sintética e simbólica. A base de conhecimento analítica é o conjunto das atividades intensivas em pesquisa e desenvolvimento, enquanto a base de conhecimento sintética se relaciona às atividades ligadas à solução de problemas concretos da indústria. A base de conhecimento simbólica, por sua vez, está relacionada às atividades criativas. A mensuração das bases de conhecimento pode ser feita através do levantamento de estatísticas de emprego que forneçam informações sobre a qualificação da mão de obra.

A presença de uma classe criativa (Florida, 2002) é reconhecida como fator crucial de atração de talentos para regiões e países. Mensurar o tamanho e desenvolvimento da "indústria criativa" de uma região, portanto, pode ser um bom indicador da vitalidade de um ecossistema de inovação (Nicotra et al., 2017).

Por fim, um ecossistema de inovação dinâmico requer a existência de fontes de financiamento robustas e diversas, assim há indicadores como disponibilidade de capital de risco (venture capital e private equity) em relação ao PIB e número de investidores-anjo por 100.000 habitantes.

\subsection{Ecossistemas de Empreendedorismo}

Conforme indicado anteriormente, a abordagem de Ecossistemas de Empreendedorismo dá ênfase a fatores institucionais. Assim, métricas a respeito do ambiente institucional para negócios, tais como práticas de governança corporativa, índice de percepção de corrupção, índice de liberdade econômica, índice de liberdade comercial e índice de qualidade regulatória são utilizadas por autores que desejam comparar ecossistemas em diferentes países (ver, por exemplo, Kshetri, 2014). Este autor também usa, na sua análise, indicadores qualitativos do ambiente institucional tais como discursos de lideranças políticas, press releases de grandes corporações, políticas de incentivo à abertura de negócios, legislação sobre investimentos, proteção a falências e mercados financeiros e mudanças em normas e valores sociais ligados ao empreendedorismo.

Enquanto existem dados suficientes para mapear o ambiente institucional, ainda há carência de métricas sobre traços individuais de empreendedores que podem afetar a atividade

\footnotetext{
${ }^{2}$ Externalidades de Jacobs são benefícios econômicos relacionados à diversificação das atividades produtivas
} numa cidade ou região 
empreendedora em nível local (Audrestch e Belitski, 2016). Cowell et al. (2017) tentaram resolver isso utilizando em sua análise dados qualitativos extraídos de entrevistas e grupos focais com empreendedores e análise de redes para mensurar os relacionamentos entre organizações de apoio a empresas, empresas e indivíduos. A rede foi mapeada a partir de interações identificadas em pesquisas sobre o uso de recursos dos empreendedores e na base de dados do Twitter.

Credit et al. (2018) ao traçarem o estado da arte das métricas de ecossistemas empreendedores, observaram que a maior parte dos estudos sobre ecossistemas de empreendedorismo utiliza dados primários devido a uma carência de dados secundários sobre empreendedorismo em geral. As fontes mais usadas são: a Eurostat, que reúne as estatísticas oficiais da União Europeia, com dados sobre seus membros e dados regionais; o Global Entrepreneurship Monitor, organização sem fins lucrativos que realiza estudos sobre taxas de empreendedorismo em diversos países; a Crunchbase, que é uma base de dados sobre empresas inovadoras, financiamento e aquisições; e a InfoDev Database, uma base do Programa InfoDev do Banco Mundial, que fomenta uma rede global de incubadoras e hubs de inovação relacionados a tecnologias do clima, agronegócio e empreendedorismo digital.

Mesmo com carência de dados secundários, houve um forte crescimento de publicações sobre ecossistemas de empreendedorismo a partir de 2009, com predominância do uso das seguintes métricas: número de startups (Audrestch e Belitski, 2017); taxa de empreendedorismo, taxa de empreendedorismo inovador, taxa de empreendedorismo de alto crescimento (Bruns et al., 2017); número de indústrias representadas por startups e dados sobre startups individuais (Nylund e Cohen, 2017); firmas incubadas em redes globais de incubadoras e participantes de hubs de inovação sobre tecnologias do clima, agronegócio e empreendedores digitais (Fernandez-Fernandez et al., 2015). Credit et al. (2018) também observaram que o Fórum Econômico Mundial desenvolveu métricas para analisar ecossistemas de empreendedorismo baseado no trabalho de Isenberg (2011), porém estas medidas foram feitas em nível nacional, perdendo a perspectiva local e características sociais e culturais.

\section{3. $\quad$ Métricas para Ecossistemas de Inovação e de Empreendedorismo}

Os intermediários - ou brokers - da inovação são apontados por Sapsed et.al (2007) como tendo um papel fundamental em um ecossistema de inovação, na medida em que agem como instituições-ponte capazes de conectar os diferentes atores locais ligados à inovação. Porém, estes intermediários também fomentam o empreendedorismo. De fato, os mecanismos de geração de empreendimentos inovadores (Aranha, 2016), que incluem incubadoras de empresas, parques tecnológicos, aceleradoras e coworkings, são elementos essenciais para estimular a interação entre a infraestrutura de conhecimento e o setor produtivo no âmbito de um ecossistema de inovação, bem como promover a cultura empreendedora, fortalecendo o ecossistema de empreendedorismo. Desse modo, o mapeamento destes mecanismos, incluindo a identificação das empresas que abrigam e das redes em que estão inseridos, é um indicador relevante da estrutura de apoio do ecossistema.

A dimensão cultural é de grande relevância nos ecossistemas de inovação e nos ecossistemas de empreendedorismo. Um possível indicador de aversão ao risco é o número de firmas que realizam operações de crédito sobre o total de firmas da região; em países como o Brasil, por exemplo, onde existe alta aversão ao risco, a maioria das firmas pequenas prefere operar com 
recursos próprios, sem lançar mão de empréstimos. A razão entre abertura e fechamento de empresas, e o número de firmas que um empreendedor abre em determinado período, podem ser indicadores de percepção de fracasso, uma vez que em sociedades onde o fechamento de empresas é associado a fracasso pessoal os empreendedores tenderão a abrir menos empresas (Wallner e Menrad, 2011). Finalmente, a tolerância à diversidade pode ser medida pelo número de programas de intercâmbio existentes nas universidades e escolas técnicas participantes do ecossistema de inovação, pelo percentual de estudantes estrangeiros sobre o total de estudantes de escolas técnicas e de universidades e pelo percentual de imigrantes sobre o total de habitantes de uma região.

Por último, para acompanhar a evolução de ecossistemas de empreendedorismo e de inovação deve-se incluir algumas métricas relativas a outputs de inovação. Outras métricas relevantes são a presença de empresas de alto crescimento (Stam, 2018), i e., empresas que apresentam crescimento médio do pessoal ocupado assalariado de pelo menos $20 \%$ ao ano por um período de três anos e tem 10 ou mais pessoas ocupadas assalariadas no ano inicial de observação (IBGE, 2018). Na mesma linha, o mapeamento das startups ${ }^{3}$ criadas e que sobrevivem também constituem um bom output do ecossistema (Nicotra et al., 2017).

\section{Mensurando Ecossistemas de Inovação e de Empreendedorismo no Brasil}

As fontes de dados para as métricas mencionadas na seção 2 estão disponíveis no Brasil, porém algumas apresentam limitações. Os dados das publicações oficiais do Ministério de Ciência, Tecnologia, Informação e Comunicações (MCTIC), do Instituto Brasileiro de Geografia e Estatística (IBGE), da Pesquisa de Inovação Tecnológica do IBGE (PINTEC) e da Plataforma Lattes do Conselho Nacional de Desenvolvimento Científico e Tecnológico (CNPq) podem ser utilizados para construir as métricas mencionadas na seção 2.1. Utilizando a PINTEC é possível dimensionar os gastos das empresas em Pesquisa e Desenvolvimento e em Atividades Inovativas. A produção científica local pode ser mapeada através da Plataforma Lattes e da Biblioteca Digital Brasileira de Teses e Dissertações (BDTD) operada pelo Instituto Brasileiro de Informação em Ciência e Tecnologia (IBICT). A proporção de Mestres e Doutores por 100.000 habitantes pode ser obtida através dos currículos cadastrados na Plataforma Lattes. Por meio da PINTEC é possível verificar a proporção de pesquisadores com pós-graduação ocupados em atividades internas de $\mathrm{P} \& \mathrm{D}$ com equivalência de dedicação total nas empresas que implementaram inovações em cada estado do país. Usando os dados de grupos de pesquisa e os currículos dos pesquisadores destes grupos que constam da Plataforma Lattes é possível mapear as redes de cooperação entre universidades e empresas do país (Urti, 2017). O INPI tem uma base de dados de patentes que permite fazer pesquisas não apenas sobre patentes concedidas no país como também sobre patentes concedidas no exterior.

No que se refere às limitações, elas variam de acordo com a base de dados. A Plataforma Lattes é alimentada de forma voluntária e, portanto, pode estar incompleta. A PINTEC só abarca empresas do setor industrial, com exceção de alguns poucos segmentos do setor de serviços, e só considera empresas com mais de 10 empregados, limitando assim seu alcance analítico. Já os dados sobre volume e percentual de investimento público e privado em $\mathrm{P} \& \mathrm{D}$ são

\footnotetext{
${ }^{3}$ Aqui consideramos como startups as empresas que buscam desenvolver um produto/serviço inovador, de base tecnológica, que tenha um modelo de negócio facilmente replicado e possível de escalar sem aumento proporcional dos seus custos (Abstartups, 2018).
} 
disponibilizados pelo MCTIC apenas em nível nacional, prejudicando análises em escala subnacional.

Indicadores de complexidade podem ser encontrados na plataforma Data Viva ${ }^{4}$ que disponibiliza informações, dados, visualizações e o indicador de complexidade econômica, desde a esfera municipal e regional até estadual e nacional. Já o cálculo da entropia pode ser feito com base nas estatísticas de atividades econômicas, que no Brasil constam da base da Relação Anual de Informações Sociais (RAIS). Como o indicador de entropia, tem por base a classificação padrão da indústria, há limitações na explicação das conexões entre os setores (Neffke et al., 2011). Outras limitações são os dados do indicador, geralmente, empregos formais da indústria, dados de patentes, produção ou exportação e importação. Este último é o grande entrave para o indicador de complexidade econômica, pois a partir da compreensão das cadeias globais de valor, os dados de comércio internacional não conseguem refletir com precisão o quanto de conhecimento os lugares possuem. Além disso, a ênfase nos dados da indústria é limitadora, pois deixa de fora uma grande quantidade de atividades que compõem a economia e agregam conhecimento.

No que se refere às bases de conhecimento, é possível utilizar os dados da RAIS utilizando os dados sobre emprego de nível superior nas atividades econômicas que compõem as diversas bases. Quanto aos dados sobre financiamento, no Brasil, o levantamento acerca do patrimônio comprometido em capital de risco é realizado por universidades (FGV), consultorias (KPMG) e bancos (BNDES), porém, somente em âmbito nacional (A importância..., 2017). O mapeamento de Investidores-Anjo pode ser feito com auxílio da rede Anjos do Brasil, que possui núcleos regionais em nove estados (Anjos do Brasil, 2019).

O volume e os tipos de investimento em inovação do Banco Nacional de Desenvolvimento Econômico e Social (BNDES), da Financiadora de Estudos e Projetos (FINEP), do CNPq, todos federais, podem ser acessados pelos portais de transparência de cada órgão. Através destes instrumentos é possível verificar os investimentos em cada estado e município. Em relação aos órgãos regionais, tais como as Fundações de Amparo a Pesquisa (FAPs) estaduais e outras agências de fomento, o detalhamento dos recursos investidos em inovação depende da existência de um mecanismo de transparência específico a cada órgão. Verifica-se, entretanto, que as agências regionais sofrem com a fragilidade ou até mesmo inexistência de mecanismos de acesso aos dados de investimentos realizados.

Sobre cultura e criatividade, desde 2014 a Federação das Indústrias do Rio de Janeiro (FIRJAN) realiza um "Mapeamento da Indústria Criativa no Brasil", calculando o "PIB criativo" e trazendo dados detalhados de emprego e remuneração dos diferentes setores - núcleo e de apoio - da indústria criativa nos 26 estados brasileiros. A última edição disponível é de fevereiro de 2019 e traz os dados relativos ao triênio 2015-2017.

Os dados sobre mecanismos de apoio podem ser encontrados através do sítio da Associação Nacional de Entidades Promotoras de Empreendimentos Inovadores ${ }^{5}$. Em nível regional, verifica-se a existência de redes estaduais destas entidades em alguns estados do país que disponibilizam as informações dos seus associados em seus sítios eletrônicos. Outros

\footnotetext{
${ }^{4}$ Criada pelo governo de Minas Gerais, a Agência de Promoção de Investimento e Comércio Exterior de Minas Gerais (INDI), com apoio da Fundação de Amparo à Pesquisa do Estado de Minas Gerais (FAPEMIG), em parceria com o MIT Media Lab. Ver http://dataviva.info/pt/

${ }^{5}$ http://anprotec.org.br/site/sobre/associados-anprotec/
} 
intermediários relevantes são os núcleos de inovação tecnológica e os escritórios de transferência de tecnologia localizados nas ICTs. O mapeamento destes atores deve ser feito a partir do sítio de cada ICT, no entanto, os indicadores destes núcleos e escritórios não são facilmente acessíveis.

A PINTEC, apesar de suas limitações, oferece um bom panorama da performance inovativa das empresas em nível nacional e regional. O mapeamento das Empresas de Alto Crescimento (EAC) é realizado pelo IBGE anualmente e publicado nas estatísticas de empreendedorismo ${ }^{6}$. $\mathrm{O}$ estudo permite dimensionar a evolução do número de EAC nos diferentes estados. Não obstante, este indicador é insuficiente, pois, não necessariamente tais empresas são inovadoras. Seria necessário identificar as EAC que são de fato inovadoras. Este dado, contudo, não está disponível em bases de dados oficiais.

Em relação aos dados sobre startups, em virtude da própria novidade do tema, ainda não existem muitos levantamentos sistemáticos disponíveis. Cabe destacar, no entanto, o levantamento realizado pela Associação Brasileira de Startups (ABStartups), que realizou em conjunto com a Accenture uma radiografia do Ecossistema Brasileiro de Startups em 2017. O estudo foi feito através de um questionário online aberto que contou com a participação de mais de 1.000 startups de todo o país. Além de mapear uma série de características e obstáculos das startups brasileiras, o estudo também calculou o índice de densidade de startups ${ }^{7}$ por estado e por cidade, bem como o índice de eficiência na geração de startups $^{8}$ em nível estadual e municipal (Abstartups, 2018). A continuidade deste levantamento e a consolidação da metodologia podem servir como importantes métricas para a caracterização de ecossistemas de inovação e de empreendedorismo no país.

Os dados do Global Entrepreneurship Monitor estão disponíveis no Brasil no site do Instituto Brasileiro de Qualidade e Produtividade, parceiro brasileiro da organização. Entretanto, a última pesquisa disponível é a de 2016, e desde então o Brasil mergulhou numa crise que pode ter afetado as taxas de empreendedorismo. Já os dados do programa do Banco Mundial InfoDev, citados por Credit et al. (2018), cobrem apenas determinadas atividades empreendedoras. Uma busca sobre o Brasil no site da InfoDev levou a informações pontuais sobre programas de apoio à agricultura familiar, apoio a incubadoras e a empreendedores digitais. Já os dados da Crunchbase contemplam cidades brasileiras, podendo ser uma boa fonte de informações sobre startups.

Os dados sobre o ambiente institucional brasileiro podem ser encontrados em estatísticas de organizações internacionais e nacionais como Heritage Foundation, Transparência Internacional e Instituto Brasileiro de Governança Corporativa (IBGC). A dificuldade de usar estes dados está relacionada à baixa disponibilidade de informações sobre como os índices utilizados foram construídos.

\section{Considerações finais}

\footnotetext{
${ }^{6}$ Entratanto, a última edição com resultados publicados é de 2015.

${ }^{7}$ Este índice foi baseado no cálculo: [número de startups respondentes] / [número de habilidades por estado ou cidade], utilizando os dados de população estimada pelo IBGE (2017).

${ }^{8}$ Este índice foi baseado no cálculo: [PIB por cidade ou estado] / [número de startups respondentes], utilizando os dados do PIB pelo IBGE (2015).
} 
A caracterização, mensuração e análise da evolução de ecossistemas de inovação e de empreendedorismo não é uma tarefa simples, uma vez que ainda não há um consenso sobre a própria definição destes conceitos e de suas dimensões e que em muitos casos os dados necessários para compor os indicadores e métricas não estão disponíveis com facilidade. Como vimos nas seções anteriores, a mensuração de ecossistemas no Brasil esbarra em alguns obstáculos relacionados às fontes de dados e disponibilização dos dados. O Quadro 2 apresenta uma síntese das métricas de ecossistemas de inovação e de empreendedorismo identificados, das fontes de dados e das principais limitações identificadas.

Quadro 2: Síntese das métricas de ecossistemas de inovação e de empreendedorismo no Brasil

\begin{tabular}{|c|c|c|c|c|}
\hline $\begin{array}{c}\text { Fonte dos } \\
\text { Dados } \\
\end{array}$ & Ecossistema & Descrição & $\begin{array}{l}\text { Nível de } \\
\text { análise }\end{array}$ & Métrica \\
\hline PINTEC & $\begin{array}{l}\text { Ecossistema de } \\
\text { inovação }\end{array}$ & $\begin{array}{l}\text { Estatísticas sobre } \\
\text { inovação }\end{array}$ & Nacional & $\begin{array}{c}\text { Taxa de empresas } \\
\text { inovadoras; redes de } \\
\text { inovação. Gastos com } \\
\text { P\&D das empresas } \\
\text { inovadoras }\end{array}$ \\
\hline IBGE & $\begin{array}{l}\text { Ecossistema de } \\
\text { empreendedorismo } \\
\text { e ecossistema de } \\
\text { inovação }\end{array}$ & $\begin{array}{c}\text { Estatísticas de } \\
\text { Empreendedorismo }\end{array}$ & Nacional & $\begin{array}{l}\text { Proporção de empresas de } \\
\text { alto crescimento em } \\
\text { relação ao total, número e } \\
\text { tipos de empresas de alto } \\
\text { crescimento }\end{array}$ \\
\hline MCTIC & $\begin{array}{l}\text { Ecossistema de } \\
\text { inovação }\end{array}$ & $\begin{array}{l}\text { Dados sobre gastos de } \\
\text { P\&D }\end{array}$ & Nacional & $\begin{array}{c}\text { Gastos de P\&D em } \\
\text { relação ao PIB; } \\
\text { Crescimento dos gastos de } \\
\text { P\&D }\end{array}$ \\
\hline FINEP & $\begin{array}{l}\text { Ecossistema de } \\
\text { inovação }\end{array}$ & $\begin{array}{l}\text { Mapa de Inovação, } \\
\text { Relatórios FNDCT }\end{array}$ & Nacional & $\begin{array}{l}\text { Empresas e instituições } \\
\text { financiadas pela Finep ao } \\
\text { longo dos últimos anos, } \\
\text { separadas por setor, e } \\
\text { relatórios sobre } \\
\text { financiamento à inovação }\end{array}$ \\
\hline BNDES & $\begin{array}{l}\text { Ecossistema de } \\
\text { inovação }\end{array}$ & $\begin{array}{l}\text { Dados sobre projetos } \\
\text { de apoio à inovação }\end{array}$ & Nacional & $\begin{array}{c}\text { Número de Startups e de } \\
\text { empresas inovadoras } \\
\text { financiadas }\end{array}$ \\
\hline RAIS & $\begin{array}{l}\text { Ecossistema de } \\
\text { inovação }\end{array}$ & $\begin{array}{l}\text { Dados sobre emprego } \\
\text { de nível superior nas } \\
\text { atividades econômicas }\end{array}$ & Nacional & $\begin{array}{l}\text { Indicador de Entropia; \% } \\
\text { de pessoal empregado em } \\
\text { atividades de C\&T }\end{array}$ \\
\hline INPI & $\begin{array}{l}\text { Ecossistema de } \\
\text { inovação }\end{array}$ & Dados sobre patentes & Nacional & $\begin{array}{c}\text { Patentes concedidas, } \\
\text { patentes triádicas por } \\
\text { milhão de pessoas }\end{array}$ \\
\hline IBGC & $\begin{array}{l}\text { Ecossistema de } \\
\text { Empreendedorismo }\end{array}$ & $\begin{array}{l}\text { Dados sobre } \\
\text { governança } \\
\text { corporativa } \\
\end{array}$ & Nacional & $\begin{array}{c}\text { Índice de governança } \\
\text { corporativa }\end{array}$ \\
\hline $\begin{array}{l}\text { Transparência } \\
\text { Internacional }\end{array}$ & $\begin{array}{c}\text { Ecossistema de } \\
\text { Empreendedorismo }\end{array}$ & Dados sobre corrupção & Internacional & $\begin{array}{l}\text { Ranking da corrupção nos } \\
\text { países }\end{array}$ \\
\hline GEM & $\begin{array}{l}\text { Ecossistema de } \\
\text { empreendedorismo }\end{array}$ & $\begin{array}{c}\text { Taxas de } \\
\text { empreendedorismo }\end{array}$ & Internacional & $\begin{array}{c}\text { Indicador de } \\
\text { Oportunidades, } \\
\text { Capacidades, Taxa de } \\
\text { falha, intenções } \\
\text { empreendedoras, } \\
\end{array}$ \\
\hline
\end{tabular}




\begin{tabular}{|c|c|c|c|c|}
\hline & & & & $\begin{array}{c}\text { atividades } \\
\text { empreendedoras em } \\
\text { estágio inicial, índice } \\
\text { motivacional, expectativa } \\
\text { de criação de emprego }\end{array}$ \\
\hline InfoDev & $\begin{array}{c}\text { Ecossistema de } \\
\text { empreendedorismo }\end{array}$ & $\begin{array}{l}\text { Atividades } \\
\text { empreendedoras }\end{array}$ & Internacional & $\begin{array}{l}\text { Série de indicadores sobre } \\
\text { Comércio, Investimento, } \\
\text { Inovação, Economia e } \\
\text { setores }\end{array}$ \\
\hline $\begin{array}{c}\text { Heritage } \\
\text { Foundation }\end{array}$ & $\begin{array}{l}\text { Ecossistema de } \\
\text { empreendedorismo }\end{array}$ & Ambiente Institucional & Internacional & $\begin{array}{c}\text { Índice de Liberdade } \\
\text { Econômica }\end{array}$ \\
\hline Crunchbase & $\begin{array}{c}\text { Ecossistema de } \\
\text { empreendedorismo }\end{array}$ & $\begin{array}{l}\text { Dados sobre empresas } \\
\text { inovadoras }\end{array}$ & Internacional & $\begin{array}{l}\text { Startups: Investimento, } \\
\text { financiamento, aquisições, } \\
\text { mercado. }\end{array}$ \\
\hline
\end{tabular}

Fonte: Elaboração Própria (2019)

Em relação aos dados disponíveis em fontes oficiais são observados alguns obstáculos para a construção de indicadores e métricas, com destaque para: i) a defasagem temporal entre a coleta e a disponibilização dos dados; ii) fontes de dados que são alimentadas voluntariamente, podendo levar à subnotificação de informações; iii) dados antigos, em virtude de pesquisas que são realizadas em intervalos de tempo grandes, p. ex. Censo, ou de levantamentos que não foram feitos recentemente, p. ex. Censo dos Grupos de Pesquisa do CNPq; iv) falta de transparência no nível regional, i. e., dados que dependem de mecanismos de transparência ou de compilação por parte de agências regionais; v) dados disponíveis somente no nível nacional; e vi) dados incompletos ou enviesados em nível regional em razão da natureza amostral do levantamento a exemplo da PINTEC.

Também são verificados obstáculos na construção de indicadores que dependem de dados não facilmente disponíveis ou até mesmo inexistentes por meio de fontes oficiais. Neste caso, as dificuldades estariam relacionadas à necessidade de coleta dos dados em fontes primárias e, posteriormente, à estruturação destes dados. Em nível regional este trabalho pode ser feito pelas fundações de estatística regionais, no entanto, muitos estados não possuem tais instituições ou as mesmas estão esvaziadas. Outro entrave constatado é o fato de que alguns dados são muito recentes e, por enquanto, dependem de associações de classe para serem levantados.

\section{Referências Bibliográficas}

A IMPORTÂNCIA do capital de risco para a inovação. BNDES, 3 de abril de 2017. Disponível em: https://www.bndes.gov.br/wps/portal/site/home/conhecimento/noticias/noticia/capital-derisco Acesso em 29/05/2019.

ABSTARTUPS - ASSOCIAÇÃO BRASILEIRA DE STARTUPS. O momento da startup brasileira e o futuro do ecossistema de inovação, 2018.

ANJOS DO BRASIL. Anjos pelo Brasil - Núcleos Regionais. Disponível em: http://www.anjosdobrasil.net/nuacutecleos-regionais.html Acesso em 29/05/2019.

ARANHA, J. A. Mecanismos de geração de empreendimentos inovadores. Mudanças na organização e na dinâmica dos ambientes e o surgimento de novos atores. ANPROTEC Tendências. Brasília, DF: ANPROTEC, 2016.

AUDRESTCH, D.B; BELITSKI, M. Entrepreneurial ecosystems in cities: establishing the framework conditions. Journal of Technology Transfer. No 42, 2017, p.1030-1051 
BAHRAMI, H.; EVANS, S. Flexible Re-Cycling and High-Technology Entrepreneurship. California Management Review 37: 62-89, 1995.

BAUMOL, W. J. Entrepreneurship: Productive, Unproductive, and Destructive. Journal of Political Economy, v. 98, n. 5, p. 893-919, 1990,

BOSCHMA, R.; IAMMARINO, S.. Related variety, trade linkages, and regional growth in Italy. Economic geography, v. 85, n. 3, p. 289-311, 2009.

BRUNS, K.; BOSMA, N.; SANDERS, M.; SCHRAMM, M. Searching for the existence of entrepreneurial ecosystems: A regional cross-section growth regression approach. Small Business Economics, No. 49 Vol.1, 2017, p. 31-54.

CASTALDI, C.; FRENKEN, K.; LOS, B. Related variety, unrelated variety and technological breakthroughs: an analysis of US state-level patenting. Regional studies, v. 49, n. 5, p. 767-781, 2015

COWELL, M; HILL, S.L; TATE, S. It takes all kinds: understanding diverse entrepreneurial ecosystems. Journal of Enterprising Communities: People and Places in the Global Economy Vol. 12 No. 2, 2018, p. 178-198

CARAYANNIS, E.; GOLETSIS, Y.; GRIGOROUDIS, E. Composite innovation metrics: MCDA and the quadruple innovation helix framework. Technological Forecasting and Social Change. Volume 131, June 2018.

CREDIT, K; MACK, E.A; MAYER, H. State of the field: Data and metrics for geographic analyses of entrepreneurial ecosystems. Geography Compass. No 12, 2018, p. 1-22

Data Viva: disponível em < http://dataviva.info/pt/> acesso em setembro de 2019.

DAWKINS, R. O Gene Egoísta. São Paulo: Companhia das Letras, 2007.

DUBINI, P. The influence of motivations and environment on business start-ups: Some hints for public policies. Journal of Business Venturing 4: 11-26, 1989.

FELD, B. Startup Communities: building an entrepreneurial ecosystem in your city. Hoboken: NJ, Wiley, 2012.

FERNÁNDEZ FERNÁNDEZ, M. T., BLANCO JIMÉNEZ, F. J., CUADRADO ROURA, J. R. Business incubation: Innovative services in an entrepreneurship ecosystem. The Service Industries Journal, Vol. 35 No 14, 2015, p. 783-800

FLORIDA, R. The Rise of the Creative Class and How it's Transforming Work, Leisure, Community and Everyday Life. New York: The Perseus Books Group, 2002.

FREEMAN, C. Technology policy and economic policy: Lessons from Japan. London: Pinter, 1987.

FRENKEN, K. Innovation, Evolution, and Complexity Theory, Edward Elgar, Cheltenham, UK and Northampton, MA, USA 2006.

FRENKEN, K.; VAN OORT, F.; VERBURG, T. Related variety, unrelated variety and regional economic growth. Regional studies, v. 41, n. 5, p. 685-697, 2007.

FURTADO, B. A.; SAKOWSKI, P. A. M.; TÓVOLLI, M. H.. Modelagem de sistemas complexos para políticas públicas. Modelagem de sistemas complexos para políticas públicas. Brasília, IPEA, 2015

GOMES, K, A, V.; FACIN, A. L. F; SALERNO, M. S.; IKENAMI, R. K. Unpacking the innovation system construct: Evolution, gaps and trends. Technological Forecasting \& Social Change, 2016. Disponível em: http://dx.doi.org/10.1016/jtechfore.2016.11.019 
HAUSSMANN, R.; HIDALGO, C. The observatory of economic complexity. Disponível em: $<$ http://atlas.media.mit.edu > acesso em setembro de 2019.

HAUSMANN, R. et al. The Atlas of Economic Complexity: Mapping paths to prosperity. 2. ed. Cambridge, Massachusetts USA: MIT Press, 2013. 71 p. ISBN 978-0-262-52542-8. Disponível em: http://atlas.cid.harvard.edu. Acesso em: 29 maio 2019.

IBGE - INSTITUTO BRASILEIRO DE GEOGRAFIA E ESTATÍSTICA. Demografia das empresas e estatísticas de empreendedorismo: 2016. Departamento de Estudos e Pesquisas/Informação Econômica, nº 31, IBGE, 2018.

ISENBERG, D. J. The big idea: How to start an entrepreneurial revolution. Harvard Business Review, 2010.JACKSON, D. D. What is an Innovation Ecosystem, 2011. Disponível em: http://erc-assoc.org/sites/default/files/topics/policy_studies/DJackson_Innvoation

Ecosystem_03-15-11.pdf

KSHETRI, N. Developing successful entrepreneurial ecosystems: Lessons from a comparison of an Asian tiger and a Baltic tiger. Baltic Journal of Management, Vol. 9 No. 3, 2014, p. 330356

LAFUENTE, E.; YANCY, V.; RIALP, J. Regional Differences in the Influence of Role Models: Comparing the Entrepreneurial Process of Rural Catalonia. Regional Studies, 41 779795, 2007.

LIGUORI, E; BENDICKSON, J; SOLOMON, S; MCDOWELL, W.C. Development of a multidimensional measure for assessing entrepreneurial ecosystems. 2018. Disponível em: htpps://doi.org/10.1080/08985626.2018.153744

LUNDVALL, B. A. National Systems of innovation: Towards a theory of innovation and interactive learning. Pinter, Londres, 1992.

MASON, C.; BROWN, R. Entrepreneurial ecosystems and growth oriented entrepreneurship. Background paper prepared for the Workshop organised by the OECD LEED Programme and the Dutch Ministry of Economic Affairs on Entrepreneurial Ecosystems and Growth Oriented Entrepreneurship, The Hague, Netherlands, 2014.

MOORE, J.F. Predators and prey: the new ecology of competition. Harvard Business Review $71(3)$, p. $75-86$

NEFFKE, F.; HENNING, M.; BOSCHMA, R. How do regions diversify over time? Industry relatedness and the development of new growth paths in regions. Economic geography, v. 87, n. 3, p. 237-265, 2011.

NELSON, R. Economics from an Evolutionary Perspective. In: NELSON, R; DOSI, G; HELFAT, C.E.; PYKA, A; WINTER, S; SAVIOTTI, P.P; LEE, K; MALERBA, F; DOPFER, $\mathrm{K}$;. Modern Evolutionary Economics - An Overview. Cambridge: Cambridge University Press, 2018. Disponível em https://www.cambridge.org/core

NEUMEYER, X., SANTOS, S. C. Sustainable Business Models, Venture Typologies, and Entrepreneurial Ecosystems: A Social Network Perspective, Journal of Cleaner Production, 2017. Disponível em: https://doi.org/10.1016/j.jclepro.2017.08.216

NICOTRA, M; ROMANO, M; DEL GIUDICE, M; SCHILLACI, C.E. The causal relation between entrepreneurial ecosystem and productive entrepreneurship: a measurement framework. Journal of Technology Transfer, 2017. Disponível em https://doi.org/10.1007/s10961-017-9628-2 
NYLUND, P. A.; COHEN, B. Collision density: driving growth in urban entrepreneurial ecosystems. International Entrepreneurship and Management, Journal, Springer, vol. 0, pages 1-20. DOI: $10.1007 / \mathrm{s} 11365-016-0424-5$

O’CONNOR, S., STAM, E.; SUSSAN, F.; AUDRESTCH, D.B. Entrepreneurial Ecosystems: The Foundations of Place-Based Renewal. In: O’Connor, S; Stam, E; Sussan, F; Audrestch, D.B (eds) Entrepreneurial Ecosystems: Place-Based Transformations and Transitions, 2018. Disponível em: https://doi.org/10.1007/978-3-319-63531-6

OH, D.S; PHILIPPS, F; PARK, S.; LEE, E. Innovation Ecosystem: a critical examination. Technovation 54, 2016, p-1-6.

PENNINGS, J. M. The urban quality of life and entrepreneurship. Academy of Management Journal 25(1): 63-79, 1982.SPIGEL, B.; HARRISON, R. Toward a process theory of entrepreneurial ecosystems. Strategic entrepreneurship journal. Special issue article, 12:151168, 2017.

SAAD, A. H.; COUETIL, N. D.; PARK, J; Technology and talent: capturing the role of universities in regional entrepreneurial ecosystems. Journal of Enterprising Communities: People and Places in the Global Economy Vol. 12 No. 2, 2018, p. 92-116

SAPSED, J.; GRANTHAM, A.; DEFILLIPI, R. A bridge over troubled waters: Bridging organisations and entrepreneurial opportunities in emerging sectors. Research Policy, $\mathrm{n}^{\circ} 36, \mathrm{pp}$. 1314-1334, 2007.

STAM, E. Measuring Entrepreneurial Ecosystems. In: O'CONNOR, A.; STAM, E.; SUSSAN, F.; AUDRETSCH, D. (Eds.). Entrepreneurial Ecosystems: Place-based Transformations and Transitions. Springer, 2018.

STAM, E.; SPIGEL, B. Entrepreneurial Ecosystems. Utretch University: School of Economics, Discussion Paper Series nr: 16-13, 2016.

THEIL, H. Statistical Decomposition Analysis. Amsterdam, North-Holland, 1972.

TORRES-FREIRE, C. Um estudo sobre os serviços intensivos em conhecimento no Brasil. In: DE NEGRI, J. A.; KUBOTA, L. C (Orgs.). Estrutura e Dinâmica do Setor de Serviços no Brasil. Brasília: IPEA, pp.107-132, 2006.

URTI, M.C.T. Interações entre o Sistema de Ciência, Tecnologia e Inovação e o Sistema de Saúde no Brasil (2000-2014). Uma análise a partir da perspectiva do conhecimento e a inovação para a inclusão social. Tese (doutorado em Políticas Públicas, Estratégias e Desenvolvimento) - Instituto de Economia, Universidade Federal do Rio de Janeiro, 2017

VAN DE VEN, A. The Development of an Infrastructure for Entrepreneurship. Journal of Business Venturing, 8: 211-230, 1993.

WALLNER, T; MENRAD, M. Extending the Innovation Ecosystem Framework. Proceedings of the XXII ISPM Conference, Hamburg, Germany. Disponível em http://www.agtil.at/uploads/images/PDFs/ISPIM_Wallner_final.pdf

VIEIRA, J. M.. Possibilidades de aplicação da análise de entropia nas Ciências Sociais e na Demografia. Ideias, v. 4, p. 52-69, 2013

XU, Z; MAAS, G. Innovation and Entrepreneurial Ecosystems as Important Building Blocks. In: MAAS, G.; JONES, P. (Eds.) Transformational Entrepreneurship Practices, 2019. Disponível em: https://doi.org/10.1007/978-3-030-11524-1_2 